\title{
VARIABLES FISIOLÓGICAS-METABÓLICAS DE ESTRÉS CALÓRICO EN VACAS BAJO SILVOPASTOREO Y PRADERA SIN ÁRBOLES ${ }^{1}$
}

\author{
Wilson Andrés Barragán-Hernández ${ }^{2}$, Liliana Mahecha-Ledesma ${ }^{3}$, Yasmin Socorro Cajas-Girón ${ }^{2}$
}

\begin{abstract}
RESUMEN
Variables fisiológicas-metabólicas de estrés calórico en vacas bajo silvopastoreo y pradera sin árboles. El presente trabajo tuvo como objetivo valorar cambios en parámetros fisiológicos y metabólicos como indicadores de estrés calórico de vacas bajo pastoreo. El estudio se llevó a cabo en el Centro de Investigación Corpoica Turipaná, Región Caribe, Cereté, Colombia, durante los años 20112012. Se determinó la temperatura ambiental (T) y la humedad relativa $(\mathrm{H})$, y en los animales: temperatura rectal (TR), temperatura de piel (TP), frecuencia respiratoria (FR) y el estatus ácido-básico. Las variables fueron medidas en la mañana $(6: 00 \mathrm{~h})$ y tarde $(13: 00 \mathrm{~h})$. Se encontró efecto del tratamiento en la temperatura ambiental con 7 y $6 \%$ menos temperatura en p-Arbus-Arbor y p-Arbor, respectivamente, comparados con el tratamiento Pasto. Hubo efecto de la hora $(\mathrm{p}<0,05)$ en $\mathrm{T}$ y $\mathrm{H}$ e interacción tratamiento $\mathrm{x}$ hora en la $\mathrm{T}(\mathrm{p}<0,05)$. Las variables TP y FR registraron efecto $(\mathrm{p}<0.05)$ del tratamiento, hora e interacción tratamiento $\mathrm{x}$ hora $(6: 00 / 13: 00 \mathrm{~h})$. Se mostró un efecto positivo del sombrío proveniente de árboles en el sistema sobre las variables fisiológicas. Los efectos negativos observados en tratamientos sin sombrío de árboles repercutieron de forma mínima en alteraciones metabólicas, evidenciando respuestas homeostáticas en el animal ante las condiciones ambientales estresantes evaluadas.
\end{abstract}

Palabras claves: bienestar animal, estrés térmico, sombra de árboles, sombra de arbustos.

\begin{abstract}
Physiological-metabolic variables of heat stress in cows grazing in silvopastoral systems and in one treeless prairie. The aim of this work was to analyze changes of physiological and metabolic parameters as indicators of heat stress of cows in pasture systems. The research was carried out from 2011 to 2012 at the Turipaná Agricultural Research Center of Corpoica located in the Caribbean region in Cereté-Colombia. Environmental temperature (T) and relative humidity $(\mathrm{H})$ were determined, as well as and rectal temperature (RT), skin temperature (ST), respiratory frequency (RF) and the acid-basic status of animals. The variables were measured in the morning $(6: 00 \mathrm{~h})$ and in the afternoon (13:00 h). Significant Statistical differences were observed $(\mathrm{p}<0.05)$ in environmental temperature treatments (T) with $7 \%$ and $6 \%$ less temperature in p-Arbus-Arbor y p-Arbor, respectively, compared with grass treatment. There was a significant hour effect on $\mathrm{T}$ and $\mathrm{H}(\mathrm{p}<0.05)$ and a significant treatment-hour interaction on $\mathrm{T}(\mathrm{p}<0.05)$. TP and FR showed a significant treatment-hour interaction per hour (6:00/13:00 h). The results show a positive effect of shadow from trees on the physiological variables. The negative effects observed on the physiological variables of unshaded treatments impacted in a minimal way the metabolic variables suggesting homeostatic responses in the animals under the evaluated stressful environmental conditions.
\end{abstract}

Keywords: animal welfare, heat stress, trees shade, shrubs shade.

\footnotetext{
Recibido: 9 setiembre, 2014. Aceptado: 19 febrero, 2015. Parte de la tesis del primer autor para obtener el título de la maestría en Ciencias Animales, Universidad de Antioquia, Colombia.

2 Corporación Colombiana de Investigación Agropecuaria (CORPOICA). Centro de Investigación Turipaná. Km 13 vía Montería - Cereté (Córd.), Colombia.wbarraganh@corpoica.org.co,ycajas@corpoica.org.co

3 Universidad de Antioquia, Facultad de Ciencias Agrarias. Grupo de Investigación en Ciencias Agrarias (GRICA) - Sistemas Sostenibles de Producción Animal. Ciudadela de Robledo, Carrera 75 N 65·87, Medellín (Ant), Colombia. Liliana.mahecha@udea.edu.co
} 


\section{INTRODUCCIÓN}

En América Latina tropical, la actividad ganadera representa el uso más importante de la tierra, siendo la actividad que más contribuye al producto interno agropecuario de los diferentes países. Dentro de esta actividad, la ganadería doble propósito tiene $78 \%$ del inventario ganadero y contribuye con el $42 \%$ de la leche producida en toda la región (Argel, 2006). En la mayoría de los predios, esta actividad se desarrolla de manera extensiva, con bajos niveles tecnológicos, lo que condiciona una baja eficiencia biológica y económica (Pérez et al., 1998).

En la región Caribe, los sistemas ganaderos de producción extensiva se caracterizan por poseer monocultivo de gramíneas y la ausencia de árboles (Navas-Panadero, 2010). Cajas-Girón y Sinclair (2001) reportan un rango de 3 a 50 árboles/ha en los predios ganaderos de esta región, indicando una baja cobertura y oferta de sombrío para los animales, lo que predispone al animal a condiciones ambientales que alteren su homeostasis térmica.

El estrés calórico, definido como la incapacidad que tiene el animal para mantener en homeostasis su temperatura corporal (Broom y Molento, 2004), se genera como respuesta biológica cuando el individuo percibe un incremento de su temperatura corporal por encima de lo normal (Morberg, 2000). En el entorno ambiental, los indicadores más relevantes para determinar estrés térmico en los animales son la temperatura del aire, la humedad relativa, la velocidad del viento y la radiación solar (Collier y Zimbelman, 2007; Arias et al., 2008).

Los principales cambios metabólicos y fisiológicos ante situaciones de estrés calórico están representados por aumento de la temperatura corporal, frecuencia respiratoria, frecuencia cardiaca, también se presenta sudoración, vasodilatación y alteración en el estatus ácido-básico en el animal (West, 2003; Arias et al., 2008; Nardone et al., 2010). Estos cambios caracterizan la respuesta ante situaciones de estrés, sin embargo, pueden presentar efectos deletéreos en la capacidad productiva y el estatus fisiológico del animal (West, 2003).

Bajo la pérdida de eficiencia para perder calor en las respuestas sensibles, se activan mecanismos de respuesta insensibles. Se ha demostrado que el aumento de la frecuencia respiratoria es un mecanismo eficiente para perder calor en situaciones de estrés calórico (Ferreira et al., 2006; Robertshaw, 2006;
Arias et al., 2008). Sin embargo, este aumento en la frecuencia respiratoria, altera la condición ácidobásica de la sangre por pérdida de $\mathrm{CO}_{2}$, reduciendo la concentración de ácido carbónico $\left(\mathrm{H}_{2} \mathrm{CO}_{3}\right)$, con el consecuente aumento de la concentración de bicarbonato $\left(\mathrm{HCO}_{3}^{-}\right)$, resultando en una alcalosis respiratoria, y posteriormente se desencadena una acidosis metabólica por sobre excreción de $\mathrm{HCO}_{3}^{-}$ (West, 2003; Nardone et al., 2010), así como también acidosis ruminal, por disminución en la cantidad de bicarbonato disponible en la saliva y su flujo hacia el rumen (Nardone et al., 2010).

Estudios relacionados con alternativas eficientes para disminuir el estrés calórico, han demostrado que el empleo de sombra o sistemas de ventilación resultan eficientes en contrarrestar los efectos deletéreos del estrés en la fisiología del animal (Armstrong, 1994; Brown-Brandl et al., 2005; Schutz et al., 2008; Tucker et al., 2008). La implementación de sistemas silvopastoriles de alta densidad, o de pastoreo en ambientes arbolados, también ha demostrado ser una alternativa eficiente para mitigar el efecto del ambiente sobre el animal, impactando positivamente la reducción de la temperatura rectal, la frecuencia respiratoria y la temperatura de piel (Souza de Abreu, 2002; Betancourt et al., 2003; Britto-Ferreira, 2010) con la ventaja de ofrecer beneficios adicionales sobre el animal y el medio ambiente (Mahecha y Angulo, 2012).

El presente trabajo tuvo como objetivo valorar cambios en parámetros fisiológicos y metabólicos como indicadores de estrés calórico de vacas bajo pastoreo.

\section{MATERIALES Y MÉTODOS}

\section{Área de estudio y diseño del experimento}

La evaluación se llevó a cabo en el Centro de Investigación Turipaná, adscrito a la Corporación Colombiana de Investigación Agropecuaria (CORPOICA), localizado en Cereté, Córdoba, Colombia ( $8^{\circ} 51^{\prime} \mathrm{N}, 75^{\circ} 49^{\prime} \mathrm{W}$, altitud de $18 \mathrm{msnm}$ ) correspondiente a la región Caribe colombiana. La zona presenta dos periodos climáticos definidos, un periodo de lluvias de mayo a noviembre y uno seco de diciembre a abril. La precipitación, promedio anual es de $1380 \mathrm{~mm}$, con una temperatura media anual de 28 ${ }^{\circ} \mathrm{C}$. Se registra una evapotranspiración potencial anual de $1240 \mathrm{~mm}$ y humedad relativa de $81 \%$ (datos de la 
estación climatológica CORPOICA-Turipaná). Según la clasificación de Holdridge (1967) la zona se clasifica ecológicamente como bosque seco tropical (bs-T).

En el año 1998 se establecieron cuatro tipos de sistemas silvopastoriles de diferente estructura y complejidad, más una pradera sin árboles (control). Para efectos de esta investigación solamente fueron usados tres tratamientos silvopastoriles compuestos por tres distintos estratos: pasturas, arbustos y árboles (productores de frutos). El tratamiento control (Pasto) estuvoconformadosolopor pasturas (Panicummaximum, Dichanthium aristatum y Cynodon nlemfuensis), el primer sistema silvopastoril presentó solamente arbustos (p-Arbus), el segundo modelo incluyó un estrato de árboles (p-Arbor) y el último modelo silvopastoril, la combinación de pasturas, arbustos y árboles (p-ArbusArbor). Los sistemas silvopastoriles de alta densidad incluyen más de 10000 arbustos por hectárea (Broom et al., 2013). Con base en este concepto, los sistemas silvopastoriles trabajados en el presente estudio fueron de baja densidad. Inicialmente las densidades utilizadas en el establecimiento fueron 625 y 159 arbustos/ha en los modelos silvopastoriles p-Arbus y p-Arbor, respectivamente. Para el caso del tratamiento p-ArbusArbor, se utilizó un método de sustitución del $25 \%$ de la población de arbustos por árboles para obtener tres estratos; en este tratamiento la densidad de arbustos fue de 468/ha, y la de árboles de 159/ha.

En el año 2004, se realizó una entresaca de especies arbóreas, disminuyendo de 159 árboles/ha (distancia de siembra de $8 \times 8 \mathrm{~m}$ ) a 39 árboles/ha. (distancia de siembra $16 \times 16 \mathrm{~m}$ ). Para el estrato arbustivo se utilizaron las especies Leucaena leucocephala y Crecentia cujete, y para el estrato arbóreo las especies fueron Guazuma ulmifolia, Cassia grandis y Albizia saman (Cuadro 1). Cada tratamiento estuvo constituido de 2 ha (100 $\mathrm{m}$ de ancho por $200 \mathrm{~m}$ de largo), para un total de 6 ha efectivas para pastoreo en las tres repeticiones.

Cuadro 1. Estructura y diversidad vegetal en tres modelos silvopastoriles y una pradera sin árboles, empleados para evaluar el estrés calórico en vacas. Centro de Investigación Corpoica Turipaná (Cereté - Córdoba, Colombia). 2012.

Table 1. Structure and diversity of plants of three silvopastoral models and one treeless prairie used to evaluate heat stress in cows. Turipaná Agricultural Research Center of Corpoica located in the Caribbean region in Cereté-Colombia. 2012.

\begin{tabular}{|c|c|c|c|}
\hline Tratamiento & Descripción & Estratos & Especies (\% composición herbácea) \\
\hline Pasto & Pastura & 1 & $\begin{array}{l}\text { Estrato herbáceo = Panicum maximum }(40 \%) \text {, Dichanthium aristatum } \\
(34 \%) \text {, Cynodon nlemfuensis }(13 \%) \text {, otras especies }(5 \%) \text { y leguminosas } \\
\text { herbáceas }(8 \%) \text {. }\end{array}$ \\
\hline p-Arbus & $\begin{array}{l}\text { Pastura }+ \text { arbustos } \\
\text { productores de hojas }\end{array}$ & 2 & $\begin{array}{l}\text { Estrato herbáceo=Panicum maximum (49\%), Dichanthium aristatum } \\
(25 \%) \text {, Cynodon nlemfuensis }(10) \text {, otras especies }(10 \%) \text { y leguminosas } \\
\text { herbáceas }(6 \%) \text {. } \\
\text { Estrato arbustivo= Leucaena leucocephala y Crecentia cujete. } \\
\text { Densidad de arbustos }=625 \text { árboles } / \text { ha. } \\
\text { Marco de siembra } 4 \mathrm{~m} \mathrm{x4} \mathrm{m.}\end{array}$ \\
\hline p-Arbor & Pasto + árboles & 2 & $\begin{array}{l}\text { Estrato herbáceo=Panicum maximum }(68 \%) \text {, otras espécies }(19 \%) \text {. } \\
\text { Estrato arbórero= Cassia gradis, Albizia saman y Guazuma ulmifolia. } \\
\text { Densidad de árboles }=39 \text { árboles } / \text { ha. } \\
\text { Distancia de siembra }=16 \mathrm{~m} \times 16 \mathrm{~m} .\end{array}$ \\
\hline p-Arbus-Arbor & Pasto + arbustos + árboles & 3 & $\begin{array}{l}\text { Estrato herbáceo = Panicum maximum }(60 \%) \text {, otras especies }(15 \%) \text {. } \\
\text { Estrato arbustivo= Leucaena leucocephala y Crecentia cujete. } \\
\text { Estrato arbóreo= Cassia gradis, Albizia saman y Guazuma ulmifolia. } \\
\text { Densidad de arbustos }=468 \text { arbustos } / \text { ha. } \\
\text { Densidad de árboles }=39 \text { árboles } / \mathrm{ha} . \\
\text { Distancia de siembra arbustos }=4 \mathrm{~m} \times 4 \mathrm{~m} . \\
\text { Distancia de siembra de árboles sustituidos por arbustos }=16 \mathrm{~m} \mathrm{x} 16 \mathrm{~m} \text {. }\end{array}$ \\
\hline
\end{tabular}




\section{Animales evaluados}

Se utilizaron dieciséis animales provenientes de un cruce Romosinuano (50\%), Holstein (25\%) y Cebú comercial $(25 \%)$ con un peso promedio de $436 \pm 64,2$ $\mathrm{kg}$, de dos y tres partos, y condición corporal entre 3,5 y 4 puntos de acuerdo con Dieter et al. (1999). La producción de leche de estas vacas varió entre 2,6 y 3,4 1/vaca/día, con $56 \pm 28$ días en lactancia y edad promedio de $52 \pm 3,9$ meses.

Los dieciséis animales fueron aleatorizados en los cuatro tratamientos evaluados, y fueron sometidos a un pastoreo rotacional en franjas de $4000 \mathrm{~m}^{2}$, con dos días de ocupación y 28 días de descanso. En cada uno de los tratamientos se ofertó sal mineralizada al $8 \%$ de fósforo y agua a voluntad.

\section{Registro de la información}

Para identificar las respuestas fisiológicas a estrés calórico, durante tres días consecutivos, se colectaron datos en la mañana a las 6:00 h (evaluación am) y en la tarde a las 13:00 h (evaluación pm), durante dos períodos de evaluación, comprendidos entre el 5 y 7 de mayo de 2011 (período 1), y del 24 al 26 de marzo de 2012 (periodo 2), con base en los trabajos de caracterización fisiológica planteados (Velasquez et al., 1996). Estos horarios presentan condiciones contrastantes de temperatura del aire y radiación solar, con menores valores registrados a las 6:00 $\mathrm{h}$ y mayores valores a las $13 \mathrm{~h}$ (Suárez et al., 2012).

Se registró la temperatura ambiental (T) y la humedad relativa $(\mathrm{H})$ en las horas de la mañana $(\mathrm{am})$ y de la tarde (pm) empleando un termohigrómetro digital portátil $\left(\mathrm{T} \pm 1{ }^{\circ} \mathrm{C} ; \mathrm{H} \pm 2 \%\right)$. El registro de la $\mathrm{T}$ y de la $\mathrm{H}$ se realizó a una altura de 1,5 metros del suelo, y en la parte media del tratamiento, con la finalidad de evitar el efecto de borde. La observación de temperatura y humedad relativa registrada fue el promedio de tres lecturas aleatorias en cada tratamiento.

Con el propósito de realizar las mediciones sobre el animal, conservando las características de cada uno de los tratamientos evaluados y asegurar un mínimo desplazamiento que pudiera afectar los parámetros fisiológicos del animal y su estatus ácido-básico, se diseñaron cuatro corrales de manejo dentro de cada uno de los tratamientos; estos fueron utilizados para confinar el animal para el registro de la información fisiológica y sanguínea al mismo tiempo, previo reposo de treinta minutos antes de cada medición. Cada corral de manejo presentó las siguientes dimensiones: $2 \mathrm{~m}$ de largo, $1,5 \mathrm{~m}$ de alto y $0,7 \mathrm{~m}$ de amplitud.

A cada animal se le registró la temperatura rectal (TR) con un termómetro clínico digital $\left( \pm 0,1^{\circ} \mathrm{C}\right)$, la temperatura de la piel (TP) mediante el uso de un termómetro infrarrojo de doble laser $\left( \pm 2,5^{\circ} \mathrm{C}\right)$ a una distancia de 1,5 metros del animal; y la frecuencia respiratoria (FR) se indicó con un fonendoscopio y un cronómetro para registrar ya fuera esta o el número de respiraciones por minuto (rpm). La TP registrada fue el producto de la media aritmética de tres lecturas obtenidas del cuello, región dorsal y anca del animal.

Se realizó una colecta de sangre mediante punción venosa en la base de la cola con tubos vacutainer heparinizados (BD Franklin Lakes NJ USA) en el horario am $/ \mathrm{pm}$, de manera paralela a la toma de información fisiológica. La sangre se analizó empleado un analizador de gases sanguíneos I-Stat 300i (Abott Point of Care Inc.), con los cartuchos de muestreo CHEM8 y G3+ (Abbott Point of Care Inc.). Cada muestra de sangre fue introducida en el cartucho usando una única jeringa de $5 \mathrm{ml}$. En los análisis sanguíneos se registraron los valores de $\mathrm{pH}, \mathrm{pCO}_{2}$, $\mathrm{HCO}_{3}, \mathrm{SO}_{2}, \mathrm{Na}, \mathrm{K}, \mathrm{Cl} \mathrm{Hct} \mathrm{y} \mathrm{Hb}$ para los horarios am/ pm. Cada muestra se analizó dentro de los quince minutos después de su colecta.

De acuerdo con los procedimientos de control, cada cartucho fue almacenado en refrigeración $\left(2-8{ }^{\circ} \mathrm{C}\right)$, y solo fueron usados antes de su fecha de expiración. Todos los análisis se realizaron empleando el analizador de gases I-Stat 300i, bajo ambiente controlado, con una temperatura entre 22 y $27{ }^{\circ} \mathrm{C}$ (Abbott Point of Care Inc.).

\section{Análisis estadístico}

Los datos colectados fueron almacenados en una hoja de cálculo de Microsoft Excel®. Para el diseño experimental se usó un arreglo de bloques completos al azar. Los períodos de muestreo como factor de bloqueo (5 al 7 de mayo de 2011, y del 24 al 26 de marzo de 2012) y cuatro animales como unidades experimentales en cada tratamiento.

Para el análisis de los datos se empleó un diseño en bloques completos al azar (DBCA) con arreglo de medidas repetidas, mediante el uso del 
procedimiento MIXED del paquete estadístico SAS (SAS Institute, 2002).

Se consideró el siguiente modelo estadístico:

$$
\begin{aligned}
& y_{i j k l m}=\mu+T_{i}+H_{j}+T H_{i j}+P_{k}+D_{l}+A(T)_{i m}+e_{i j k l m} \\
& \operatorname{con} P_{k} i d\left(N ; \sigma_{k}^{2}\right), \\
& D_{l} i d\left(N ; \sigma_{l}^{2}\right) \\
& A(T)_{i m} i d\left(N ; \sigma_{i m}^{2}\right. \\
& \text { y } e_{i j k l m} i d\left(N ; \sigma^{2}\right)
\end{aligned}
$$

Dentro del modelo fueron empleadas como variables aleatorias el efecto del período $P$ (factor de bloqueo período 1 del 5 al 7 de mayo de 2011 y período 2 del 24 al 26 de marzo de 2012), el efecto del día de evaluación $D$ y el efecto del animal $A$ dentro de cada tratamiento $T$; y como efectos fijos el tratamiento (Pasto, p-Arbus, P-Arbor y p-Arbus-Arbor), la hora de muestreo (am y pm) y la interacción entre el tratamiento y la hora de muestreo. Se consideró el período como factor de bloqueo con la finalidad de aislar los posibles efectos de cada tiempo de evaluación en las respuestas observadas. En este proceso se utilizó la estructura de varianzas y covarianzas que mejor explicó la posibilidad de correlación existente entre las medidas repetidas de un mismo animal y la heterogeneidad de varianzas en los efectos aleatorios y residuales del modelo. El modelo se escogió con base en los estadísticos AIC y BIC. Las variables T, H, SO, $\mathrm{Cl}$, HGB, HTC y K consideraron una estructura de varianzas y covarianzas no estructurada, la variable NA y TR presentó una estructura de componentes de varianza, la variable $\mathrm{pH}$ simetría compuesta y las variables FR y TP simetría compuestas y varianzas heterogéneas para efectos aleatorios y componentes de varianza para los residuos del modelo.

Para todos los casos de significancia se consideró como error alfa 0,05 y como tendencia una probabilidad entre 0,05 y 0,1 . Los efectos significativos se separaron utilizando la sentencia LSMEANS con el ajuste de Tukey.

\section{RESULTADOS Y DISCUSIÓN}

Hubo efecto significativo del tratamiento, de la hora y de la interacción tratamiento x hora sobre la temperatura ambiental $(\mathrm{T})(\mathrm{p}<0,05)$. Se encontró 5,0 y $4,5 \%$ menos temperatura en los tratamientos silvopastoriles con cobertura arbórea p-Arbus-Arbor y p-Arbor, comparados con el testigo sin árboles, con tendencia significativa en ambos casos ( $\mathrm{p}=0,06 \mathrm{y}$ 0,10 , respectivamente). Así mismo, la $\mathrm{T}$ en el sistema silvopastoril con solo arbustos (p-Arbus) fue mayor que la de los sistemas silvopastoriles que incluían árboles (p-Arbus-Arbor y p-Arbor) (Cuadro 2). Todos los tratamientos incrementaron la temperatura de

Cuadro 2. Temperatura ambiental y humedad relativa por día para tres modelos silvopastoriles y una pradera sin árboles en el centro de investigación Corpoica Turipaná (Cereté - Córdoba, Colombia) durante el período del 5 al 7 de mayo de 2011 (período 1) y del 24 al 26 de marzo de 2012 (período 2).

Table 2. Environmental temperature and relative humidity per day in three models of silvopastoral systems and one treeless prairie at the Turipaná Agricultural Research Center of Corpoica (Cerete-Córdoba, Colombia) from May $5^{\text {th }}$ to May $7^{\text {th }}, 2011$ (period 1) and from March 24 $4^{\text {th }}$ to March 26 $6^{\text {th }}, 2012$ (period 2).

\begin{tabular}{lcccccccc}
\hline Variable & Pasto & p-Arbus & p-Arbor & $\begin{array}{c}\text { p-Arbus- } \\
\text { Arbor }\end{array}$ & $\begin{array}{c}\text { Error estándar } \\
\text { de la media }\end{array}$ & $\begin{array}{c}\text { p-valor } \\
\text { Trat. }\end{array}$ & $\begin{array}{c}\text { p-valor } \\
\text { Hora }\end{array}$ & $\begin{array}{c}\text { p-valor } \\
\text { Trat. } x \text { hora }\end{array}$ \\
\hline $\begin{array}{l}\text { Temperatura } \\
\text { ambiental }\left({ }^{\circ} \mathrm{C}\right)\end{array}$ & $31,36 \mathrm{ab}$ & $31,70 \mathrm{a}$ & $29,94 \mathrm{~b}$ & $29,80 \mathrm{~b}$ & 0,69 & 0,0049 & $<, 0001$ & 0,0166 \\
$\begin{array}{l}\text { Humedad } \\
\text { relativa }(\%)\end{array}$ & 62,38 & 61,98 & 64,07 & 63,51 & 3,44 & 0,9630 & $<, 0001$ & 0,5116 \\
\hline
\end{tabular}

Letras diferentes entre promedios en la misma fila indican diferencia estadística significativa $\mathrm{p}<0,05$ / Different letters between means in the same row indicate significant statistical difference $\mathrm{p}<0,05$.

p-Arbus: pastura + arbustos productores de hojas, p-Arbor: pasto + árboles, p-Arbus-Arbor: pasto + arbustos + árboles / p-Arbus: pasture + leaf producer shrubs, p-Arbor: grass + trees, p-Arbust- Arbor: grass + shrubs+ trees. 
forma significativa al pasar del registro am al pm. La temperatura en promedio aumentó $11^{\circ} \mathrm{C}$ al pasar de la medición de $6: 00 \mathrm{~h}$ a las $13: 00 \mathrm{~h}\left(25,05\right.$ vs. $36,35^{\circ} \mathrm{C}$, respectivamente). Hubo diferencia entre tratamientos en la hora de mayor calor (pm), y se encontró una mayor temperatura ambiental $(\mathrm{p}<0,05)$ en los tratamientos sin sombra o, sin sombra proveniente de arbustos (pasto y p-Arbus, respectivamente), comparados con los tratamientos p-Arbus-Arbor y p-Arbor, los cuales aportaron sombra de árboles. Los sistemas silvopastoriles con sombra proveniente de árboles (p-Arbus-Arbor y p-Arbor), lograron reducir en promedio $3{ }^{\circ} \mathrm{C}$ la temperatura ambiental en la hora pm comparados con los primeros $(\mathrm{P}<0,05)$ (Figura 1). En México, los sistemas silvopastoriles intensivos que ofrecen sombra de árboles y arbustos, permitieron reducir en $8,6^{\circ} \mathrm{C}$ la temperatura ambiente en las horas de mayor intensidad solar, comparado con los sistemas

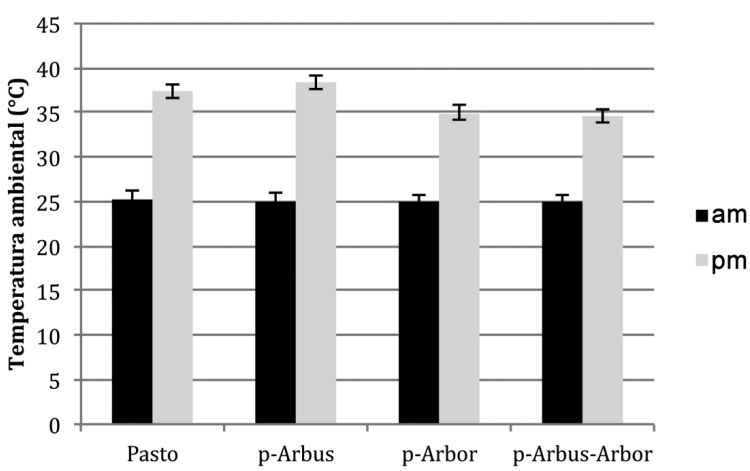

Figura 1. Temperatura ambiental am y pm en tres modelos silvopastoriles y en una pradera sin árboles. Centro de investigación Corpoica Turipaná (Cereté - Córdoba, Colombia) durante el período del 5 al 7 de mayo de 2011 (período 1) y del 24 al 26 de marzo de 2012 (período 2).

Las líneas negras sobre las barras representan el error estándar.

p-Arbus: pastura + arbustos productores de hojas, p-Arbor: pasto + árboles, p-Arbus-Arbor: pasto + arbustos + árboles.

Figure 1. Am and pm environmental temperature in three silvopastoral models of and one treeless prairie at the Turipaná Agricultural Research Center of Corpoica (Cerete-Córdoba, Colombia) from May $5^{\text {th }}$ to May $7^{\text {th }}, 2011$ (period 1) and from March $24^{\text {th }}$ to March 26 $6^{\text {th }}, 2012$ (period 2).

The black lines on the bars represent the standard error.

p-Arbus: pasture + leaf producer shrubs, p-Arbor: grass + trees, p-Arbus-Arbor: grass + shrubs + trees tradicionales sin sombrío (Solís et al., 2011). Baliscei et al. (2013) encontraron que el sistema silvopastoril disminuyó la $\mathrm{T}$ e hizo el ambiente más armonioso porque disminuyó las diferencias entre las estaciones climáticas verano e invierno.

La humedad relativa $(\mathrm{H})$ presentó cambios significativos entre la hora am y la pm en todos los tratamientos $(\mathrm{p}<0,05)$, con comportamiento contrario al de la temperatura con una reducción significativa al pasar del registro am al pm (78,60 vs. 47,37\%). Sin embargo, al sacar el promedio por tratamiento, no se encontraron diferencias significativas ni interacción tratamiento $\mathrm{x}$ hora (Cuadro 2). En Colombia se han reportado ciclos diurnos unimodales con mayores humedades entre las cuatro y siete horas y los menores valores hacia las trece y dieciséis horas, con una relación inversa entre la T y la H (Marín et al., 2004).

La TP y la FR fueron diferentes entre tratamientos $(\mathrm{p}<0,05)$ y se encontró una tendencia estadística en la TR $(\mathrm{p}=0,0549)$. La hora de evaluación afectó significativamente $(\mathrm{p}<0,0001)$ las tres variables con menores valores en la hora más fresca de evaluación (am vs pm). Sin embargo, la respuesta no fue igual en todos los tratamientos y la interacción tratamiento $\mathrm{x}$ hora resultó significativa para las tres variables $(\mathrm{p}<0,05)$ (Cuadro 3). En la TP hubo un incremento significativo del $11 \%(\mathrm{p}<0,05)$ en el tratamiento testigo sin árboles ni arbustos (pasto) y en el tratamiento que tenía solo arbustos (p-Arbus), al pasar del registro am al pm (aproximadamente $4{ }^{\circ} \mathrm{C}$ ), mientras que en el tratamiento con árboles para sombrío (p-Arbor) y en el sistema silvopastoril multiestrato con mayor sombrío (p-Arbus-Arbor) el incremento fue de solo el 2 y 5\% $\left(1\right.$ y $\left.2{ }^{\circ} \mathrm{C}\right)$, respectivamente.

Cuando se compararon todos los tratamientos a la hora de mayor calor ( $\mathrm{pm})$, el testigo sin árboles (Pasto) y el silvopastoril con solo arbustos (p-Arbus) presentaron una mayor TP $(\mathrm{p}<0,05)$ que los tratamientos silvopastoriles con sombrío proveniente de árboles (p-Arbor y p-Arbus-Arbor) (Figura 2). En la FR se encontraron diferencias significativas $(\mathrm{p}>0,05)$ entre la medición am y pm para el testigo sin árboles (pasto) y para el silvopastoril con solo arbustos (p-Arbus), los cuales mostraron claras evidencias de disconfort ambiental con aumentos del 33\%; mientras que en los sistemas silvopastoriles con árboles (p-Arbol y p-Arbus-Arbol) el incremento solo fue del 13 y 3\%, respectivamente y las diferencias entre el registro am y el pm no fueron significativas $(p>0,05)$ (Figura 2). 
Cuadro 3. Temperatura rectal, temperatura de piel y frecuencia respiratoria para tres modelos silvopastoriles y una pradera sin árboles en el centro de investigación Corpoica Turipaná (Cereté - Córdoba, Colombia) durante el período del 5 al 7 de mayo de 2011 (período 1) y del 24 al 26 de marzo de 2012 (período 2).

Table 3. Rectal temperature, skin temperature, and respiration rate in three silvopastoral models andone treeless prairie at the Turipaná Agricultural Research Center of Corpoica (Cerete-Córdoba, Colombia) from May $5^{\text {th }}$ to May $7^{\text {th }}, 2011$ (period 1) and from March 24 ${ }^{\text {th }}$ to March 26 ${ }^{\text {th }}, 2012$ (period 2).

\begin{tabular}{lccccccc}
\hline Variable & Pasto & p-Arbus & p-Arbor & $\begin{array}{c}\text { p-Arbus- } \\
\text { Arbor }\end{array}$ & $\begin{array}{c}\text { p-valor } \\
\text { trat. }\end{array}$ & $\begin{array}{c}\text { p-valor } \\
\text { hora }\end{array}$ & $\begin{array}{c}\text { p-valor } \\
\text { trat. } x \text { hora }\end{array}$ \\
\hline Temperatura rectal $\left({ }^{\circ} \mathrm{C}\right)$ & $39,07 \pm 0,37$ & $39,16 \pm 0,38$ & $38,65 \pm 0,38$ & $38,87 \pm 0,37$ & 0,0549 & $<, 0001$ & $<, 0001$ \\
Temperatura de piel $\left({ }^{\circ} \mathrm{C}\right)$ & $37,91 \pm 0,22 \mathrm{a}$ & $38,18 \pm 0,26 \mathrm{a}$ & $36,55 \pm 0,22 \mathrm{~b}$ & $36,39 \pm 0,20 \mathrm{~b}$ & 0,0003 & $<, 0001$ & 0,0017 \\
Frecuencia respiratoria (FR) & $70,82 \pm 2,66 \mathrm{a}$ & $69,57 \pm 2,78 \mathrm{ac}$ & $63,92 \pm 2,85 \mathrm{bc}$ & $61,94 \pm 2,76 \mathrm{~b}$ & 0,0107 & $<, 0001$ & 0,0038 \\
\hline
\end{tabular}

Se presentan los valores promedio \pm el error estándar / The average values are reported \pm standard error. p-Arbus: pastura + arbustos productores de hojas, p-Arbor: pasto + árboles, p-Arbus-Arbor: pasto + arbustos + árboles / p-Arbus: pasture + leaf producer shrubs, p-Arbor: grass + trees, p-Arbus-Arbor: grass + shrubs + trees.

En la hora de mayor calor (pm), los animales de todos los tratamientos sobrepasaron el límite de frecuencia respiratoria (FR) de $60 \mathrm{rpm}$ descrito por Arias et al. (2008). Sin embargo, el efecto del sombrío de árboles en los sistemas silvopastoriles p-Arbor y p-ArbusArbol fue apreciable, sobrepasando en conjunto este límite solo en el $9 \%$ mientras que en los tratamientos sin sombrío proveniente de árboles (Pasto y p-Arbus) en conjunto registraron un incremento en la FR del $34 \%$ por encima del límite mencionado.

En la hora de mayor calor (pm), los tratamientos Testigo (pasto) y con solo arbustos (p-Arbus) presentaron una mayor FR $(\mathrm{p}<0,05)$ comparados con los tratamientos con sombrío proveniente de árboles (p-Arbor y p-Arbus-Arbor) (Figura 2). La TR tuvo el mismo comportamiento de la FR con diferencias significativas $(\mathrm{p}<0,05)$ entre la medición am y pm para los tratamientos pasto y $\mathrm{p}$-Arbus y sin diferencia significativa $(\mathrm{p}>0,05)$ para los $\mathrm{p}$-Arbor y $\mathrm{p}$-ArbusArbor). En el tratamiento pasto, la TR ascendió 1 ${ }^{\circ} \mathrm{C}$ y en el p-Arbus aumentó $1,1^{\circ} \mathrm{C}$, al pasar de la medición am a pm, siendo en ambos casos significativa $(\mathrm{p}<0,05)$, lo que representó un incremento de $2,51 \%$ y $2,93 \%$, respectivamente. En la medición de mayor calor (pm), y por lo tanto, de mayor susceptibilidad a estrés calórico, los animales que se encontraban en los sistemas silvopastoriles p-Arbor y p-Arbus-Arbor tuvieron una menor TR $(\mathrm{p}<0,05)$ comparada con la de los animales de los tratamientos pasto y p-Arbus. En esta hora, la TR de los animales que se encontraban en los sistemas silvopastoriles con cobertura arbórea
(p-Arbor y p-Arbus-Arbor), en promedio, no rebasó el límite superior de $39,1^{\circ} \mathrm{C}$ descrito por Cunningham (2003) como inicio de alteración homestática de la temperatura. Contrario a esto, se observó que los tratamientos sin cobertura arbórea (pasto y p-Arbus), registraron incrementos de 0,55 y $0,72{ }^{\circ} \mathrm{C}$ en la $\mathrm{TR}$, respectivamente, sobre el límite crítico superior (Figura 2).

El efecto ambiental que se presentó sobre la alteración fisiológica de los individuos, fue reafirmado con base en las diferencias encontradas en el índice de temperatura humedad (ITH) obtenido en cada tratamiento, alcanzando valores de 81,6, 81,8, 80,3 y 80,1 para pasto, p-Arbus, p-Arbor y p-Arbus-Arbor, respectivamente, siendo más bajo en los tratamientos con cobertura arbórea. Este índice fue generado con base en los resultados de temperatura ambiental y humedad relativa (datos no presentados), siguiendo las indicaciones propuestas por Dikmen y Hensen (2009). De acuerdo con lo descrito por Amstromg (1994), St-Pierre et al. (2003) y Zimbelman et al. (2009) para ganado Holstein, un ITH $>72$ indica condiciones de estrés calórico en los animales; mientras que para ganado de carne se reporta un ITH $>75$ (St-Pierre et al., 2003; Brown-Brandl et al., 2005; Boonprong et al., 2008); y para animales $1 / 2$ Holstein - 1/2 Cebú, un ITH $>79$ puntos (De Azevedo et al., 2005).

En el presente trabajo, a pesar de las diferencias encontradas entre tratamientos, todos los valores de ITH estuvieron por encima de los valores de referencia y estarían indicando en mayor o menor 

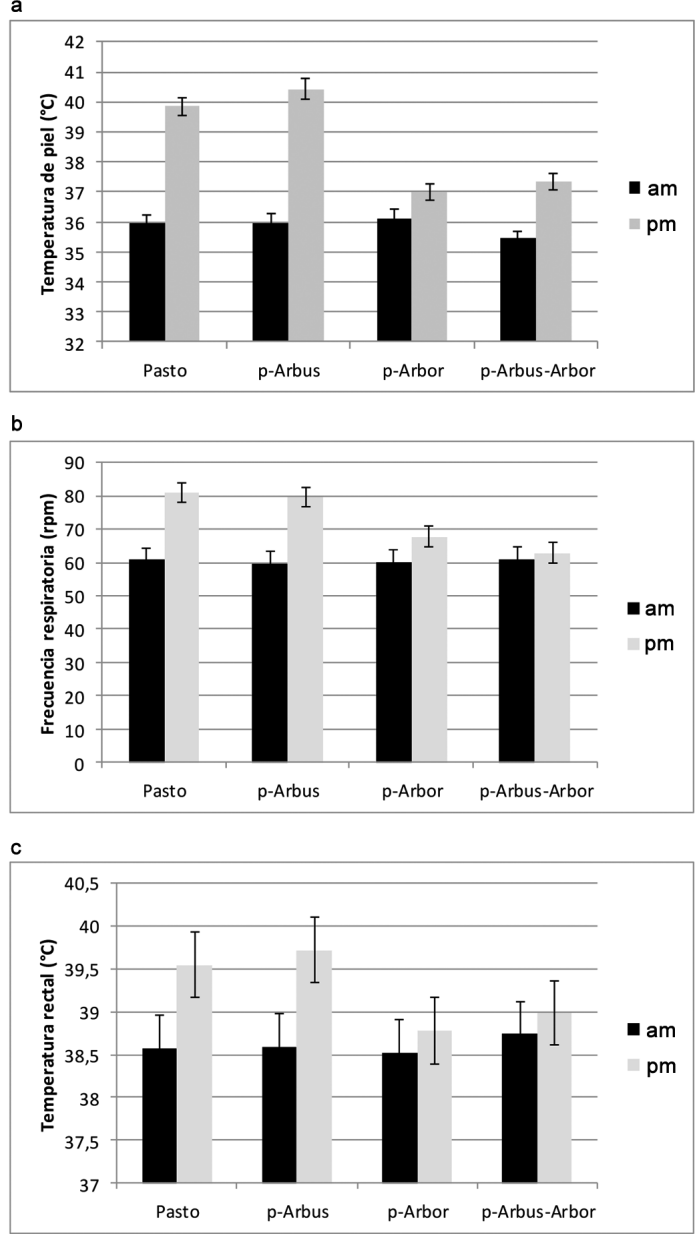

Figura 2. a) Temperatura de piel, b) frecuencia respiratoria $y$ c) temperatura rectal, evaluada en horarios am y pm en vacas, bajo tres modelos silvopastoriles y en una pradera sin árboles. Centro de Investigación Corpoica Turipaná (Cereté - Córdoba, Colombia) durante el período del 5 al 7 de mayo de 2011 (período 1) y del 24 al 26 de marzo de 2012 (período 2).

Las líneas negras sobre las barras representan el error estándar.

p-Arbus: pastura + arbustos productores de hojas, p-Arbor: pasto + árboles, p-Arbus-Arbor: pasto + arbustos + árboles

Figure 2. a) Skin temperature, b) respiration rate and c) rectal temperature, evaluated during am and pm schedules in three silvopastoral models and one treeless at the Turipaná Agricultural Research Center of Corpoica (Cerete-Córdoba, Colombia) from May $5^{\text {th }}$ to May $7^{\text {th }}, 2011$ (period 1) and from March 24 ${ }^{\text {th }}$ to March $26^{\text {th }}, 2012$ (period 2).

The black lines on the bars represent the standard error.

p-Arbus: pasture + leaf producer shrubs, p-Arbor: grass + trees, p-Arbus-Arbor: grass + shrubs + trees. grado condiciones de estrés calórico. Sin embargo, los límites propuestos, tanto para ganado de leche como para ganado de carne, fueron generados en ambientes diferentes a los referidos en el estudio. Adicionalmente, los valores de referencia de ITH desarrollados para ganado bovino, inicialmente fueron estandarizados bajo cámaras térmicas con efectos ambientales constantes, lo cual no sucede en la práctica (Collier y Zimbelman, 2007). Considerando los aspectos mencionados y dada las características genéticas de los animales usados en el presente estudio (50\% Romosinuano, 25 Hosltein y 25\% Cebú comercial), con un porcentaje de sangre que se relaciona con adaptabilidad ambiental por parte del criollo Romosinuano y el Cebú comercial, se podría pensar en la existencia de un valor de referencia superior al límite propuesto por los autores citados.

Los resultados encontrados revelan que la variación en los parámetros fisiológicos de vacas ante condiciones ambientales contrastantes del día (am $v s \mathrm{pm}$ ) en sistemas silvopastoriles no siempre es la misma y para este estudio estuvo influenciada positivamente por la presencia de sombra proveniente de árboles. Diferentes estudios citan el efecto de la sombra sintética (Armstrong, 1994; Brown-Brandl et al., 2005; Schutz et al., 2008; Tucker et al., 2008) sobre la disminución de respuestas fisiológicas al estrés calórico. Asimismo, algunos autores citan que no existe diferencia entre la sombra de árboles y la artificial (Valtorta et al., 1997). Sin embargo, el uso de árboles en sistemas ganaderos acarrea otra serie de beneficios y productos que mejoran la sostenibilidad ambiental de los sistemas de producción (CajasGiron 2002; Cajas-Giron et al., 2010). Estudios que relacionaban el comportamiento fisiológico de animales que pastorean en sistemas silvopastoriles o ambientes con alta cobertura de árboles (potreros arbolados), registraron disminución en la temperatura rectal y de piel con valores de 0,5 y $3{ }^{\circ} \mathrm{C}$, respectivamente (Souza de Abreu, 2002; Betancourt et al., 2003; BrittoFerreira, 2010). De igual forma, se ha demostrado que la sombra de los árboles contribuye a disminuir el impacto ambiental directo de la radiación solar sobre las respuestas fisiológicas insensibles, conservando parámetros como la frecuencia respiratoria dentro de los rangos normales (Souza de Abreu, 2002).

Respecto a los parámetros metabólicos, el tratamiento no presentó efecto significativo $(\mathrm{p}>0,05)$ en ninguno de ellos (Cuadro 4) y la hora solo fue 
Cuadro 4. Valores en status ácido-básico, electrolitos y metabolitos sanguíneos de vacas para tres modelos silvopastoriles y una pradera sin árboles en el Centro de Investigación Corpoica Turipaná (Cereté - Córdoba, Colombia) durante el período del 5 al 7 de mayo de 2011 (período 1) y del 24 al 26 de marzo de 2012 (período 2).

En paréntesis error estándar de la media.

Table 4. Values of acid-base status, electrolytes and blood metabolites of cows in three silvopastoral models and one treeless prairie at the Turipaná Agricultural Research Center of Corpoica (Cerete-Córdoba, Colombia) from May $5^{\text {th }}$ to May $7^{\text {th }}, 2011$ (period 1) and from March $24^{\text {th }}$ to March $26^{\text {th }}, 2012$ (period 2). Standard error of the mean in parentheses.

\begin{tabular}{lccccccc}
\hline Variable & Pasto & p-Arbus & p-Arbor & $\begin{array}{c}\text { p-Arbus- } \\
\text { Arbor }\end{array}$ & p-valor Trat & $\begin{array}{c}\text { p-valor } \\
\text { Hora }\end{array}$ & $\begin{array}{c}\text { p-valor } \\
\text { Trat x hora }\end{array}$ \\
\hline $\mathrm{pH}(\mathrm{Log})$ & $7,49(0,04)$ & $7,49(0,04)$ & $7,51(0,04)$ & $7,48(0,04)$ & 0,6744 & $0,0,0169$ & 0,0121 \\
$\mathrm{pCO}_{2}(\mathrm{~mm} \mathrm{Hg})$ & $34,71(2,75)$ & $35,94(2,83)$ & $36,49(2,81)$ & $37,30(2,77)$ & 0,4770 & 0,0062 & 0,0405 \\
$\mathrm{HCO}_{3}(\mathrm{mmol} / \mathrm{ml})$ & $26,22(0,59)$ & $26,72(0,66)$ & $28,41(0,65)$ & $27,81(0,62)$ & 0,053 & 0,7959 & 0,1704 \\
$\mathrm{Na}(\mathrm{mmol} / \mathrm{ml})$ & $137,44(1,21)$ & $139,26(1,26)$ & $138,6(1,25)$ & $138,49(1,23)$ & 0,2873 & $<, 0001$ & 0,1413 \\
$\mathrm{~K}(\mathrm{mmol} / \mathrm{ml})$ & $4,45(0,19)$ & $4,55(0,19)$ & $4,33(0,19)$ & $4,56(0,20)$ & 0,1204 & 0,9682 & 0,3877 \\
$\mathrm{Cl}(\mathrm{mmol} / \mathrm{ml})$ & $107,36(2,21)$ & $108,02(2,23)$ & $106,05(2,22)$ & $107,31(2,22)$ & 0,4021 & $<, 0001$ & 0,6784 \\
$\mathrm{Hemoglobina}(\mathrm{g} / \mathrm{dl})$ & $6,98(0,34)$ & $7,11(0,37)$ & $7,02(0,37)$ & $6,37(0,35)$ & 0,3106 & 0,0805 & 0,8448 \\
\hline
\end{tabular}

p-Arbus: pastura + arbustos productores de hojas, p-Arbor: pasto + árboles, p-Arbus-Arbor: pasto + arbustos + árboles / p-Arbus: pasture + leaf producer shrubs, p-Arbor: grass + trees, p-Arbus-Arbor: grass + shrubs + trees.

significativa $(\mathrm{p}<0,05)$; mientras $\mathrm{pH}, \mathrm{pCO}_{2}, \mathrm{Na}$ y Cl. Sin embargo, la variable pH registró una interacción significativa $(\mathrm{p}<0,05)$ de los factores tratamiento y hora, y la variable; mientras $\mathrm{pCO}_{2}$ registró una leve interacción, indicando respuestas diferentes en los tratamientos entre el horario am-pm (Cuadro 4). En el $\mathrm{pH}$ hubo incremento en todos los tratamientos al pasar del registro am al pm, excepto en el sistema silvopastoril multiestrato (p-Arbus-Arbor), en el que se registró disminución. No obstante, la variación solo fue significativa $(\mathrm{p}<0,05)$ en el tratamiento testigo.

En la $\mathrm{pCO}_{2}$ hubo una respuesta contrastante a la del $\mathrm{pH}$ con disminución en todos los tratamientos al pasar del registro am al pm, excepto en el sistema silvopastoril multiestrato (p-Arbus-Arbor) en el que se registró incremento, sin diferencias significativas en ninguno de los tratamientos $(\mathrm{p}<0,05)$ (Cuadro 5). La literatura no referencia trabajos que evalúen el efecto de ambientes silvopastoriles sobre el estrés calórico determinado por cambios en el estatus ácidobásico y electrolitos sanguíneos. Sin embargo, se reporta un aumento de la frecuencia respiratoria como mecanismo de pérdida de calor (Robertshaw, 2006), lo cual altera la homeostasis ácido-básica por aumento de la excreción de $\mathrm{CO}_{2}$, con un consecuente aumento en el pH sanguíneo, normalmente caracterizado como alcalosis respiratoria (West, 2003; Beatty et al., 2006; Nardone et al., 2010). La excreción de $\mathrm{CO}_{2}$, se produce por la hiperventilación generada en el aumento de la frecuencia respiratoria, y la disociación del $\mathrm{H}_{2} \mathrm{CO}_{3}$ en $\mathrm{H}_{2} \mathrm{O}$ y CO 2 (Srinkandakumar y Jhonson, 2004), con lo cual se aumenta la relación $\mathrm{HCO}_{3} / \mathrm{H}_{2} \mathrm{CO}_{3}$ generando un incremento del pH sanguíneo (Cunningham, 2003). De acuerdo con las referencias mencionadas, se esperaría que bajo condiciones de estrés calórico se presente un aumento en el pH sanguíneo, disminución del $\mathrm{pCO}_{2}$ y aumento en $\mathrm{HCO}_{3}$, con una alcalosis respiratoria. Bajo este análisis, en el presente trabajo, hubo manifestaciones metabólicas de estrés con aumentos de $\mathrm{pH}$ y disminución de $\mathrm{pCO}_{2}$ al pasar del registro am al pm en todos los tratamientos menos en el silvopastoril multiestrato (p-Arbus-Arbor) en donde se encontró disminución de $\mathrm{pH}$ y aumento de $\mathrm{CO}_{2}$, siendo estadísticamente claras solo para el $\mathrm{pH}$ en el testigo. Sin embargo, se podría decir que solo fueron manifestaciones iniciales de estrés calórico que pudieron ser sorteadas a través de los mecanismos de termorregulación ya que no desencadenaron en una alcalosis respiratoria con alteración del $\mathrm{CO}_{2}, \mathrm{HCO}_{3} \mathrm{O}$ de las demás variables metabólicas evaluadas.

Cuando se presentan cambios en el $\mathrm{pH}$, sin llegar a cruzar los rangos de alcalosis y acidosis, 
Cuadro 5. Interacción entre tratamiento x hora de muestreo para los valores de $\mathrm{pH}$ y presión parcial de $\mathrm{CO}_{2}$ en vacas en tres modelos silvopastoriles y una pradera sin árboles en el Centro de Investigación Corpoica Turipaná (Cereté - Córdoba, Colombia) durante el período del 5 al 7 de Mayo de 2011 (período 1) y del 24 al 26 de marzo de 2012 (período 2).

En paréntesis error estándar de la media.

Table 5. Interaction between treatment $\mathrm{x}$ hour of sampling for $\mathrm{pH}$ and partial pressure of $\mathrm{CO}_{2}$ in three silvopastoral models and one treeless prairie at the Turipaná Agricultural Research Center of Corpoica (Cerete-Córdoba, Colombia) from May $5^{\text {th }}$ to May $7^{\text {th }}, 2011$ (period 1) and from March $24^{\text {th }}$ to March $26^{\text {th }}, 2012$ (period 2).

Standard error of the mean in parentheses.

\begin{tabular}{lcccccccc}
\hline Variable & Pasto & \multicolumn{3}{c}{ p-Arbus } & \multicolumn{3}{c}{ p-Arbor } & \multicolumn{3}{c}{ p-Arbus-Arbor } \\
\cline { 2 - 9 } & $\mathbf{a m}$ & $\mathbf{p m}$ & $\mathbf{a m}$ & $\mathbf{p m}$ & $\mathbf{a m}$ & $\mathbf{p m}$ & $\mathbf{a m}$ & $\mathbf{p m}$ \\
\hline $\mathrm{pH}(\mathrm{Log})$ & $7,47 \mathrm{a}$ & $7,52 \mathrm{~b}$ & $7,49 \mathrm{ab}$ & $7,49 \mathrm{ab}$ & $7,48 \mathrm{ab}$ & $7,53 \mathrm{~b}$ & $7,49 \mathrm{ab}$ & $7,47 \mathrm{ab}$ \\
& $(0,035)$ & $(0,036)$ & $(0,037)$ & $(0,037)$ & $(0,037)$ & $(0,037)$ & $(0,036)$ & $(0,036)$ \\
$\mathrm{pCO}_{2}(\mathrm{~mm} \mathrm{Hg})$ & $36,08(2,8)$ & $33,3(2,8)$ & $36,89(2,9)$ & $34,99(2,9)$ & $38,45(2,9)$ & $34,54(2,9)$ & $36,69(2,8)$ & $37,92(2,8)$ \\
\hline
\end{tabular}

Letras diferentes entre promedios en la misma fila indican diferencia estadística significativa $\mathrm{p}<0,05$ / Different letters between means in the same row indicate significant statistical difference $\mathrm{p}<0.05$.

p-Arbus: pastura + arbustos productores de hojas, p-Arbor: pasto + árboles, p-Arbus-Arbor: pasto + arbustos + árboles / p-Arbus: pasture + leaf producer shrubs, p-Arbor: grass + trees, p-Arbus-Arbor: grass + shrubs + trees.

promovidos por cambios en la $\mathrm{pCO}_{2}$ o en el $\mathrm{HCO}_{3}$, se pudo asegurar que la respuesta animal mediada por los mecanismos Buffer en el metabolismo, controlaron las alteraciones severas del pH (Beatty et al., 2006). Uno de los mecanismos que regulan los procesos de alcalosis respiratoria es la excreción de $\mathrm{HCO}_{3}$, en la orina mediada por la función renal (Cunningham, 2003; West, 2003), aumentando los valores de $\mathrm{pH}$ en la orina (Kadzere et al., 2002; Beatty et al, 2006; Nardone et al., 2010). Estudios que evalúan cambios en la condición ácido-básica por efecto del calor, indicaron ausencia de efecto significativo entre las condiciones frías o calientes en las concentraciones de $\mathrm{HCO}_{3}$ sanguíneo en bovinos, evaluadas en un único período del día (Srinkandakumar y Johnson, 2004), tal como se reportó en este estudio.

En resumen, los sistemas silvopastoriles con sombra proveniente de árboles (p-Arbus-Arbor y el p-Arbor) presentaron el mejor registro de las variables fisiológicas evaluadas, mientras que el testigo y el sistema p-Arbus presentaron alteración negativa, tanto al pasar del registro am al pm como al comparar los tratamientos en la hora pm. Estas alteraciones fisiológicas solo se vieron reflejadas en alteración significativa del $\mathrm{pH}$ en el tratamiento testigo al pasar del registro am al pm. Estos resultados indican que las alteraciones encontradas cuando los animales pastoreaban en pasturas sin árboles, no fueron lo suficientemente agudas para determinar cambios en el estatus ácido-básico y en los electrolitos sanguíneos, evidenciando respuestas homeostáticas en el animal sobre los factores de estrés ambiental (Collier y Zimbelman, 2007). La respuesta encontrada puede estar relacionada con la capacidad de tolerancia al estrés calórico del grupo genético utilizado en la evaluación, sugiriendo una alta eficiencia de los animales en mantener la homeostasis metabólica y en la adaptación a las condiciones de trópico bajo. Está documentado que el grupo genético Romosinuano, a pesar de ser un animal Bos taurus, presenta alta tolerancia a las condiciones de estrés calórico (Johnson et al., 2012) por poseer la piel suelta, las piernas más largas, orejas cortas, pelaje corto y brillante (Da Silva et al., 2007) y baja tasa de crecimiento, lo que traduce en una baja tasa metabólica (Scharf et al., 2010). De igual forma, Hansen (2004) reporta los animales cebú, como individuos capacitados evolutivamente para afrontar ambientes calurosos, dada las características de la piel, la cantidad y distribución de las glándulas sudoríparas y la baja tasa metabólica.

Contrario a lo esperado, el tratamiento silvopastoril que incluía pasto y arbustos, pese a ser uno de los sistemas silvopastoriles que presentó mejor comportamiento productivo (datos no presentados), tuvo un comportamiento similar al tratamiento testigo en las variables evaluadas. Estos resultados 
podrían atribuirse en parte, a las prácticas de manejo relacionadas con el control de crecimiento en los arbustos, lo cual limitó la posibilidad de sombra difusa en estos con su consecuente beneficio térmico en los animales, tal como lo reporta Ceballos et al. (2011) en sistemas silvopastoriles de alta densidad de leucaena.

Estudios futuros deberían considerar evaluaciones con grupos genéticos termo-tolerantes y termosensibles, en períodos más prolongados de muestreo $\mathrm{y}$ en variables productivas, de calidad de producto $\mathrm{y}$ reproductivas, con la finalidad de profundizar en la bondad de los sistemas silvopastoriles en la mitigación del efecto ambiental sobre la homeostasis térmica de los animales y su relación con la eficiencia productivareproductiva-económica y de bienestar animal, buscada en los sistemas de producción animal en el nuevo contexto mundial.

\section{AGRADECIMIENTOS}

Los autores agradecen al Proyecto "Valoración integral de los beneficios de sistemas silvopastoriles de estratos múltiples sobre la sostenibilidad del suelo y la productividad animal en el sistema doble propósito de la región Caribe" financiado por el Ministerio de Agricultura y Desarrollo Rural (MADR) y ejecutado por Corporación Colombiana de Investigación Agropecuaria (CORPOICA), y al proyecto de Sostenibilidad 2011-2012 (Comité para el Desarrollo de Investigación (CODI), Universidad de Antioquia).

\section{LITERATURA CITADA}

Argel, P.J. 2006. Contribución de los forrajes mejorados a la productividad ganadera en sistemas de doble propósito. Arch. Latinoam. Prod. Anim. 14:65-72.

Arias, R., T. Mader, y P. Escoba. 2008. Factores climáticos que afectan el desempeño productivo del ganado bovino de carne y leche. Arch. Med. Vet. 40:7-22.

Armstrong, D. 1994. Heat stress interaction with shade and cooling. J. Dairy Sci. 77:2044-2050.

Baliscei, M.A, O.R. Barbosa, W de. Souza, M.A. Costa, K. A. Teixeira, and E. De O. Queiroz. 2013. Microclimate without shade and silvopastoral system during summer and winter. Acta Scientiarum. Animal Sci. 35:49-56.

Beatty, D.T., A. Barnes, E. Taylor, D. Pethick, M. McCarthy, and S.K. Maloney. 2006. Physiological responses of Bos taurus and Bos indicus cattle to prolonged, continuous heat and humidity. J. Anim. Sci. 84:972-985.

Betancourt, K., M. Ibrahim, C. Harvey, y B. Vargas. 2003. Efecto de la cobertura arbórea sobre el comportamiento animal en fincas ganaderas de doble propósito en Matiguás, Matagalpa, Nicaragua. Agroforestería en las Américas 10(39-40):47-51.

Boonprong, S., A. Choothesa, C. Sribhen, N. Parvizi, and C. Vajrabukka. 2008. Productivity of Thai Brahman and Simmental-Brahman crossbred (Kabinburi) cattle in central Thailand Int. J. Biometeorol. 52:409-15.

Britto-Ferreira, L. 2010. Respostas fisiológicas e comportamentais de bovinos submetidos a diferentes ofertas de sombra Dissertação (mestrado) - Universidade Federal de Santa Catarina, Centro de Ciências Agrárias, Programa de Pós-Graduação em Agroecossistemas, BRA.

Broom, D., e C. Molento. 2004. Bem-estar animal: conceito e questões relacionadas-revisão. Arch. Vet. Sci. 9(2):1-11.

Broom, D.M., F.M. Galindo, and E. Murgueitio. 2013. Sustainable, efficient livestock production with high biodiversity and good welfare for animals. Proc. R. Soc. B. 280:20132025. doi http://dx.doi.org/10.1098/ rspb.2013.2025

Brown-Brandl, T., R. Eigenberg, J. Nienaber, and G.L. Hahn. 2005. Dynamic response indicators of heat stress in shaded and non-shaded feedlot cattle, Part 1: Analyses of indicators. Biosyst. Eng. 90:451-462.

Cajas-Girón, Y.S. 2002. Impacts of tree diversity on the productivity of silvopastoral systems in seasonally dry areas of Colombia. $\mathrm{PhD}$ thesis, University of Wales, Bangor, GBR.

Cajas-Giron, Y., and F. Sinclair. 2001. Characterization of multistrata silvopastoral systems on seasonally dry pastures in the Caribbean Region of Colombia. Agroforestry Syst. 53:215-225.

Cajas-Girón, Y.S., J. Martínez, B. Panza, A. Mogollón, y W. Barragan. 2010. Valoración integral de los beneficios de sistemas silvopastoriles de estratos múltiples sobre la sostenibilidad del suelo y la productividad animal en el Sistema doble propósito de la región Caribe. Corporación Colombiana de Investigación Agropecuaria - CORPOICA. Ministerio de Agricultura y Desarrollo Rural, COL.

Ceballos, M.C., C. Cuartas, J.F. Naranjo, J.E. Rivera, F. Arenas, E. Murgueitio, y A.M. Tarazona. 2011. Efecto de la temperatura y la humedad ambiental sobre el comportamiento de consumo en sistemas 
silvopastoriles intensivos y posibles implicaciones en el confort térmico. Rev. Colomb. Cienc. Pecu. 24:368.

Collier, R.J., and R.B. Zimbelman. 2007. Heat stress effects on cattle: what we know and what we don't know. In: Proc. of the Southwest Nutrition and Management Conference, The University of Arizona, Tucson, February 23rd. http://ag.arizona.edu/ans/ swnmc/Proceedings/2007/Collier_2007SWNMC.pdf (accessed 1 sep. 2014).

Cunningham, J. 2003. Fisiología veterinaria. Ed. Elsevier, ESP.

Da Silva, R., D. Façanha, and M. Guilhermino. 2007. Evaluation of thermal stress index for dairy cows in tropical regions. Rev. Bras. de Zootec. 36:1192-1198.

De Azevedo M., M. Ávila, H Mattana, Â. Quintão, I. Barboza, J. Neves, e L. Esteves. 2005. Estimativa de níveis críticos superiores do índice de temperatura e umidade para vacas leiteiras $1 / 2,3 / 4$ e $7 / 8$ HolandêsZebu em lactação. R. Bras. Zootec. 34:2000-2008.

Dieter H., T. Díaz, y H. Flórez. 1999. Guía para la evaluación de la condición corporal de vacas doble propósito. CORPOICA. Programa Nacional de Nutrición Animal, Bogotá, COL.

Dikmen, S., and P. Hansen. 2009. Is the temperaturehumidity index the best indicator of heat stress in lactating dairy cows in a subtropical environment? J. Dairy Sci. 92:109-116.

Ferreira, F., M. Pires, M. Martinez, S. Coelho, A. Carvalho, P. Ferreira, E. Facury Filho, e W. Campos. 2006. Parâmetros fisiológicos de bovinos cruzados submetidos ao estresse calórico. Arq. Bras. Med. Vet. Zootec 58:732-738.

Johnson J.S., B. Scharf, P. Weaber, E. Eichen, and D. Spiers. 2012. Patterns of heat response and adaptation on summer pasture: A comparison of heat-sensitive (Angus) and - tolerant (Romosinuano) cattle. J. Therm. Biol. 37:344-350.

Hansen, P. 2004. Physiological and cellular adaptations of zebu cattle to thermal stress. Anim. Reprod. Sci. 82:349-360

Holdridge, L.R. 1967. Life zone ecology. Tropical Science Center, San José, CRC.

Kadzere, C., M. Murphy, N. Silanikove, and E. Maltz. 2002. Heat stress in lactating dairy cows: a review. Livest. Prod. Sci. 77:59-91.

Mahecha, L., and J. Angulo. 2012. Nutrient management in silvopastoral systems for economically and environmentally sustainable cattle production: a case study from Colombia. In: J. Whalen, editor, Soil fertility omprovement and integrated nutrient Management - A Global Perspective. InTech, CRO. p. 201-216.

Marín, L.M., G. Poveda, y H.A. Moreno. 2004. Análisis del ciclo diurno de la humedad relativa en 17 aeropuertos de Colombia. Comunicación presentada en: XVI Seminario Nacional de Hidráulica e Hidrología Sociedad Colombiana de Ingenieros Sociedad de Ingenieros del Quindío Universidad del Quindío Corporación Autónoma Regional del Quindío Armenia 29, 30 y 31 de octubre de 2004.

Morberg, G. 2000. Biological response to stress: implication for animal walfare. In: G. Morberg, and J. Mench, editors, The biology of animal stress: basic principles and implications for Animal welfare. Cabi Publishing, GBR. p. 1-19.

Nardone, A., B. Ronchi, N. Lacetera, M.S. Ranieri, and U. Bernabucci. 2010. Effects of climate changes on animal production and sustainability of livestock systems. Livest. Sci. 130:57-69.

Navas-Panadero, A. 2010. Importance of silvopastoral systems on caloric stress reduction in tropical livestock productions. Rev. Med. Vet. 19:113-122.

Pérez, J., G. Martínez, L. Albarado, y G. Ossa. 1998. Características productivas, reproductivas y biofísicas de cuatro fincas en el sistema doble propósito en el Departamento de Córdoba. En: S. Reza, editor, La Investigación Pecuaria. CORPOICA, COL. p. 21-27.

Robershaw, D. 2006. Mechanisms for the control of respiratory evaporative heat loss in panting animals. J. Appl. Physiol. 101:664-668.

SAS Institute Inc. 2002. SAS/STAT User's guide, v. $64^{\text {th }}$ ed. SAS Inst., Cary, NC, USA.

Scharf B., J.A. Carroll, D.G. Riley, C.C. Chase, S.W. Coleman, D.H. Keisler, R.L. Weaber, and D.E. Spier. 2010. Evaluation of physiological and blood serum differences in heat-tolerant (Romosinuano) and heat-susceptible (Angus) Bos taurus cattle during controlled heat challenge. J Anim Sci. 88:2321-2336.

Schutz, K. E., N.R. Cox, and L.R. Matthews. 2008. How important is shade to dairy cattle? Choice between shade or lying following different levels of lying deprivation. App. Anim. Beha. Sci. 114:307-318.

Solís, G.F., J. Ku Solorio, y M. Barros. 2011. Comportamiento ingestivo y bienestar animal en sistemas silvopastoriles del trópico Michoacano. Memorias III Congreso sobre sistemas silvopastoriles intensivos, para la ganadería sostenible del siglo XXI. Fundación 
Produce Michoacán, COFRUPO, SAGARPA, UADY (Universidad Autónoma de Yucatán), Morelia, MEX.

Souza de Abreu, M.H. 2002. Contribution of trees to the control of heat stress in dairy cows and the financial viability of livestock farms in humid tropics. $\mathrm{PhD}$. Thesis, CATIE, Turrialba, CRC.

St-Pierre, N., V. Covanob, and G. Schnitkey. 2003. Economic losses from heat stress by US livestock industries. J. Dairy Sci. 86(E. Suppl.):E52-E77.

Srikandakumar, A., and E. Johnson. 2004 . Effect of heat stress on milk production, rectal temperature, respiratory rate and blood chemistry in Holstein, Jersey and Australian Milking Zebú cows. Trop. Anim. Health Prod. 36:686-692.

Suárez, E., S. Reza, E. Díaz, F. García, I. Pastrana, H. Cuadrado, y M. Espinosa. 2012. Efectos de las condiciones ambientales sobre el comportamiento ingestivo en bovinos de carne en un sistema intensivo en el Valle del Sinú. Rev. Corpoica 13:207-212.

Tucker, C.B., A.R. Rogers, and K.E. Schutz. 2008. Effect of solar radiation on dairy cattle behaviour, use of shade and body temperature in a pasture-based system. App. Anim. Beha. Sci. 109:141-154.

Valtorta, S.E., P.E. Leva, and M.R. Gallardo. 1997. Evaluation of different shades to improve dairy cattle well-being in Argentina. Int J Biometeorol. 41:65-67.

Velasquez, J., H. Florez, y J. Cardozo. 1996. Respuesta fisiológica al estrés por calor de vacas criollas Sanmartinero. En: S.X. Pulido, y C.A. Jaramillo, editores, Agrociencia y tecnología del siglo XXI. CORPOICA, Orinoquia, COL. p. 248-250.

West, J.W. 2003. Effects of heat-stress on production in dairy cattle. J. Dairy Sci. 86:2131-44.

Zimbelman, R.B., R.P. Rhoads, M.L. Rhoad, G.C. Duff, L.H. Baumgard, and R.J.Collier. 2009. ARe-evaluation of the impact of temperature humidity index (THI) and black globe humidity index (BGHI) on milk production in high producing dairy cows. Western Dairy Management Conference, Reno (Nevada). http://animal.cals.arizona. edu/swnmc/Proceedings/2009/14Collier_09.pdf (accessed 10 nov. 2013). 
